\title{
Use of computer assisted orthopedic surgery in pelvic and acetabular trauma
}

\author{
Amr Abdelgawad ${ }^{1}$, Ralitsa Akins², Enes M. Kanlic ${ }^{3}$ \\ ${ }^{1}$ Department of Orthopedic Surgery \\ and Rehabilitation, Paul L. Foster School \\ of Medicine \\ ${ }^{2}$ Advanced Teaching and Assessment \\ in Clinical Simulation, Paul L. Foster \\ School of Medicine \\ ${ }^{3}$ Department of Orthopedic Surgery \\ and Rehabilitation, Paul L. Foster School \\ Computer assisted orthopedic surgery (CAOS) is a recent concept in \\ orthopedics. Its use in orthopedic trauma is becoming more popular. \\ Pelvic and acetabular trauma is one of the applications where CAOS \\ can play an important role to facilitate the surgery. In this review ar- \\ ticle, we provide an overview of the structure of CAOS with special \\ emphasis on its role in pelvic and acetabular trauma. The use of CAOS \\ has many advantages in the field of orthopedic trauma, however, many \\ obstacles are still present that prevent its wide use.
} of Medicine

Corresponding author:

Enes M. Kanlic, MD, FACS

Department of Orthopedic Surgery and Rehabilitation

Texas Tech University Health

Science Center

Paul L. Foster School of Medicine

4801 Alberta Ave

El Paso, TX 79905

USA

enes.kanlic@ttuhsc.edu

Tel.: + 19155456852

Fax: + 19155456704

Received: 31 December 2010

Accepted: 1 October 2011

Copyright (C) 2011 by

Academy of Sciences and Arts

of Bosnia and Herzegovina.

E-mail for permission to publish:

amabih@anubih.ba
Key words: Pelvis, Fractures, Osteosynthesis, Computer navigation.

\section{Introduction}

Computer assisted orthopedic surgery (CAOS) is a new concept in orthopedic surgery. In orthopedics, CAOS has been most extensively used in arthroplasty (1-7) and to a less extent in spine surgery $(8,9)$. CAOS in arthroplasty allows for defining patient anatomy (e.g. acetabular abduction and flexion, hip offset and length) and recreating it more precisely and with less outliers than conventional methods of reconstruction (1-7). CAOS in spine surgery allows for more accurate insertion of pedicle screws, especially in cases of spine deformity and scoliosis, in which the normal anatomy of the spine is altered and finding the pathway of the pedicle screw becomes more difficult (8, 9). Recently, there has been a growing interest in using CAOS in orthopedic trauma (10). In 1998, the National Institute of Health/American Association of Orthopedic Surgeons Workgroup on Technology Transfer in Orthopedics suggested that orthopedic trauma may become one of the fields where image-guided surgery might have an important role (11).

In orthopedic trauma, CAOS has been introduced to improve the accuracy of placement of the implants in 
treating pelvis and acetabular fractures. The complex anatomy of this region requires significant use of fluoroscopy to guide the insertion of the implants, i.e. screws.

\section{The components of CAOS}

A CAOS navigation system consists of a computerized navigation unit (which has the procedure-specific software) connected to an optical tracking camera; trackers (light emitting diode - LED) which are reference guides attached to the surgical site, LED attached to the image source (C-arm), LED attached to the surgical instruments ("smart") and a monitor $(12,13)$ (Figure 1). The process of "image guided navigation" has three elements:

Data acquisition images of the patient anatomy / pathology (fracture) are entered into the computer and these images are used to guide the surgeon to perform the intended procedure. In trauma surgery, data acqui- sition is usually performed by one of three imaging sources (conventional 2D fluoroscopy, 3D fluoroscopy, CT scans, sees below).

Registration the process of relating the collected data of the patient's three-dimensional anatomy / fracture (from the data acquisition step) to the patient's actual position and anatomy on the surgical field. This is done by the computerized navigation unit identifying the LED tracker attached to the patient's body. In cases of pelvic and acetabular trauma, the LED trackers are usually inserted into the iliac crest. Surgical tools with a tracker attached to them are also registered by the navigational system.

Tracking provides feedback during surgery by identifying the relative position of the LED attached to the bone and surgical tools. The navigation unit tracks the position of the surgical tools in relation to the patient's anatomy and the surgical instruments are displayed on the monitor, superimposed on the patient imaging study, as

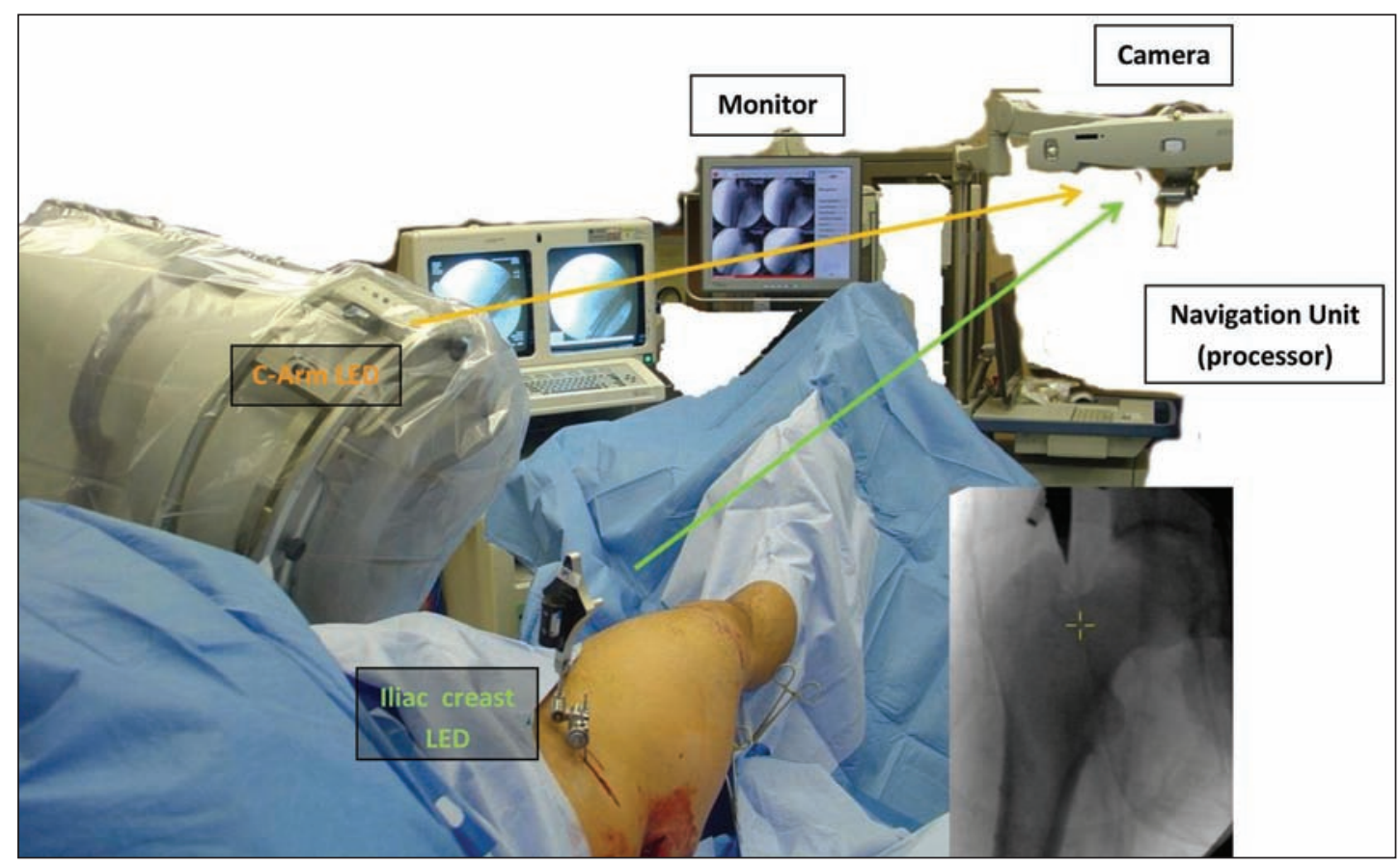

Figure 1 Composition of CAOS: LED trackers attached to the iliac crest, LED attached to C-arm, optical tracking camera, computerized navigation unit, monitor 
continuous virtual real time navigation. This will give feedback to the surgeon about the relative position of his instrument or his implant. The surgeon can use this feedback to adjust the position of the instrument or the implant relative to the patient's anatomy, to follow the correct anatomical pathway (12). There are mainly three types of data acquisition in trauma surgery:

- Intra-operative Conventional 2D Fluoroscopy Two-D (dimensional) fluoroscopy is the most popularly used method of data collection in trauma surgery, including pelvic and acetabular trauma. The C-arm is widely available in the majority of hospitals, and the amount of radiation generated by the C-arm is less than CT scans (computer tomogram) and 3D fluoroscopy (see below). The disadvantage of conventional 2D fluoroscopy lies in the decreased delineation of anatomic structures, especially in anatomically complex regions (e.g. the pelvis and acetabulum).

- Intra-operative 3D Fluoroscopy This new technology allows 3D reconstruction based on multiple intra-operative fluoroscopy images. During 3D fluoroscopy there is increased patient exposure to ionizing radiation, with additional exposure(s) if the operating field has to be broadened or if the reduction has to be checked or repeated. With the Iso- $\mathrm{C}^{\circledast}$ (Siemens, Germany) device, a multi-planar reconstruction for each field $(12 \times 12 \times 12 \mathrm{~cm})$ is calculated from $100 \mathrm{C}$ arm images.). 3D has improved image quality compared to 2D fluoroscopes and better availability compared to CT. The use of 3D fluoroscopy based navigation is becoming more popular nowadays (e.g. acetabular and calcaneal surgeries) $(14,15)$.

- Computerized Tomography Computerized tomography is used for precise fracture delineation in spine, acetabulum or pelvic operations. It has the advantage of high accuracy for anatomy identification. There are two types of CT acquisition: pre-operative CT and intra operative CT.

- Pre operative CT The major disadvantage of using pre operative CT in trauma surgery is the necessity for absolute fracture stability, so the relationship between the bone fragments may be sustained throughout the period from the preoperative CT acquisition to the time of surgery, i.e. stable pelvic or acetabular fractures $(16,17)$. The matching procedure (registration) in pelvic and acetabular trauma can be difficult, because the pelvis is surrounded by abundant soft tissue (14).

- Intra-operative CT This eliminates the disadvantage of multiple imaging for registration because registration can be done at the same time as data acquisition. Another advantage of intraoperative $\mathrm{CT}$ is that it decreases the risk of displacement of the fracture between the time of CT acquisition and surgery. Intra operative CT is more commonly used in percutaneous sacroiliac fixation or acetabular fixation $(18,19)$. Howev$\mathrm{er}$, it requires performing the procedure in the CT suite or having a CT machine in the operating theatre. It is an expensive option, not available in many places, and irradiation of the patient is higher compared with $\mathrm{C}$-arm usage.

\section{Sacral and iliosacral screw insertion}

Most sacral fractures and iliosacral joint disruptions are now being treated by closed reduction and percutaneous screw fixation. Due to the very narrow safe corridor for these screws, it is necessary to ensure the correct pathway for the screw. Guide wires are inserted first and then after checking that these wires are in the desired place, cannulated screws are threaded over the guide wires. To obtain the correct pathway, the guide wires have to be in the correct di- 
rection in three views of the sacrum (inlet, outlet and lateral). Using conventional fluoroscopy for insertion of these screws is not a simple task. After it is done in one plane, the C-arm needs to be moved to the other planes to check the position of the implant. The C-arm has to continue shifting between the three positions (a large amount of irradiation for the patient and the surgeon) throughout the whole procedure. Multiple attempts are usually required before achieving the correct pathway as the surgeon can only assess the pathway in one plane at a time. The perfect trajectory may be obtained in one view and when the other view is checked, the guide wire may be in an incorrect position. Also with false passages, the guide wire may encroach over the nerve foramens and cause nerve palsy or vascular damage (20-22). To improve precision, decrease the radiation, and shorten the surgery time CAOS should be used when inserting sacral and iliosacral screws.

Stöckle et al. (14) inserted 28 iliosacral screws (27 in S1 and 1 in S2) by image guided navigation. The authors had 27 of 28 screws (96\%) well placed as assessed by postoperative radiographs and CT scan (14). In another report, CAOS was associated with accurate placement of 21 out of a total of 22 implanted iliosacral screws in 10 patients $(9$ patients with trauma and one with instability), where one screw perforated the anterior wall of the sacrum and had to be revised; this deviation of the screw was attributed to the bending of the navigated guide during implantation. The authors recommended using a navigated sleeve rather than a navigated power driver, as this may lead to less bending of the guide pin (19). Schep et al. (23) treated 24 patients with post partum pelvic pain syndrome. Each patient had 2 screws inserted bilaterally ( 96 screws). They compared 48 iliosacral screws inserted with fluoroscopy based navigation with 48 screws inserted by conventional fluoroscopy. The fluoroscopy time in the navigation group was 0.7 minutes versus 1.8 minutes in the conventionally treated group. There was one case in the navigation group that developed a post-operative S2 sensory deficit (23). It is important to note that their study was performed on patients with no fractures. In cases of fractures, it is mandatory that the fracture parts do not move after loading the fluoroscopic images into the navigation system. If the fracture site has moved after reduction (which is not common with sacral fractures), the virtual pictures on the navigation monitor will not represent the actual reality and registration must be repeated. Other authors have also used navigation for insertion of sacroiliac screws with similar results $(24,25)$.

\section{Acetabular and pelvic fixation}

Percutaneous fixation of the posterior and anterior column of the acetabulum has become more popular recently $(21,26)$. Multiple fluoroscopy views (iliac, obturatory, inlet, outlet, iliac-inlet view and obturatoroutlet views) are needed to obtain the correct pathway and avoid complications (e.g. penetration of the joint space, neuro-vascular injury) and secure fixation (optimal screw orientation and screw length).

To date there have been no studies comparing the use of conventional fluoroscopy with CAOS in inserting percutaneous anterior and posterior column screws. Rosenberg et al. (18) used image guided navigation in inserting eight percutaneous screws in five patients with minimally displaced acetabular fractures and the result was optimal placement of 6 out of 8 screws; the bending of the guide wire was considered responsible for the 2 incorrect screw placements. Crowl and Kahler (2002) reported the use of image-guided navigation in performing minimally invasive fixation of 9 anterior column fractures of the acetabulum and this resulted 
in safe placement of all screws (27). Image guided navigation in acetabular fractures has been described by other studies (level III or IV) with similar good results $(14,24,25)$.

\section{Our method of applying CAOS in pelvic and acetabular fracture}

After positioning the patient on the table, closed reduction is obtained (or maintained) using traction and manipulation. The trackers (LED) are attached to the iliac wing bone. Appropriate radiographs are obtained after achieving the closed reduction. For sacral, sacroiliac and pelvic injuries: anteroposterior pelvis, lateral sacral, inlet and outlet views are necessary. Acetabular injuries require: anteroposterior pelvis, obturatory and iliac views. For percutaneous anterior or posterior column screws: we add the obturator-outlet view and iliac-inlet view for better assessment of the pathway of the guide wire and ensure that it does not penetrate the hip joint or violate the cortex (28).

Those images are related by software to the patient's anatomy using a reference (patient tracker attached to iliac wing). The in-

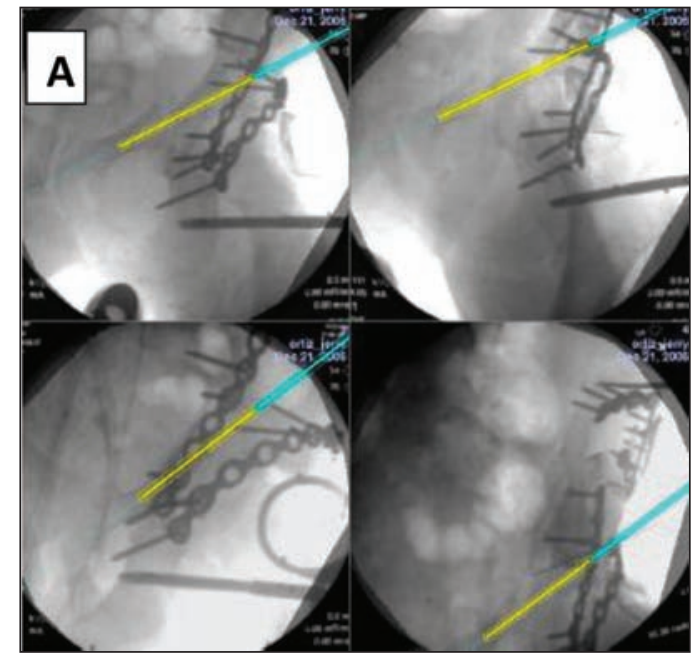

struments (e.g. guide sleeve) are registered. The C-arm machine is moved away from the operating table and we operate observing the monitor of the navigation unit, which shows the four saved and needed views of the patient's anatomy.

The pathway for the guide wire of the cannulated screw (which is controlled by the registered sleeve guide) will be visible on the screen in relationship with each of four needed images (virtual reality). This allows us to continuously check the position of our guide wire. There is no need to repeat the radiographs or move the $\mathrm{C}$-arm from one position to the other. The software has the option of extending the trajectory from the bone surface towards the desired position calculating the length and thickness of the screw. Final radiographs are taken at the end of the procedure to ensure that the virtual reality on the monitor is identical to the actual position of the screw (Figure 2).

\section{Advantages of using navigation in pelvic and acetabular trauma}

The reduction of radiation exposure (compared to the use of conventional fluoros-

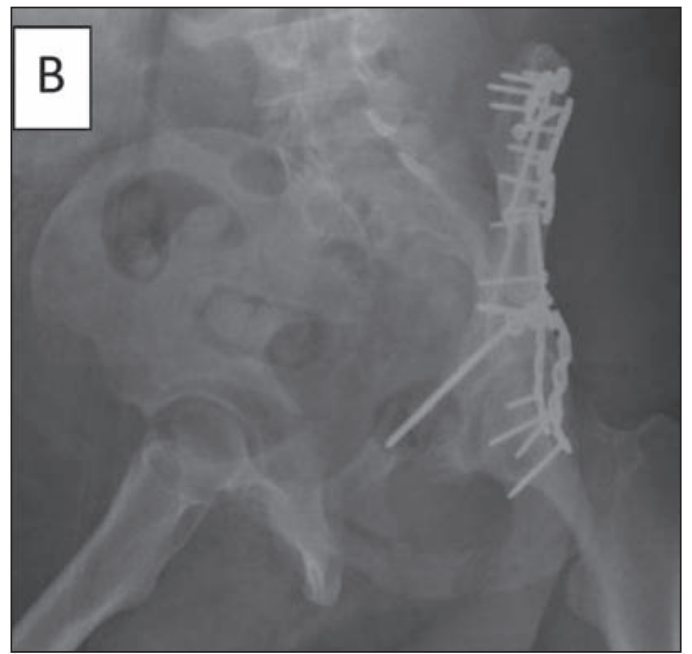

Figure 2 After fixing the acetabular fracture from the posterior approach, CAOS is used to insert the percutaneous anterior column screw. (A) Four views are shown on the monitor of the navigation unit with a virtual image of the guide wire trajectory on these views. (B) Final radiograph 
copy) is one of the main advantages of using the CAOS. CAOS can show the surgeon continuous virtual reality images of the instruments and implants, with no need to repeat the fluoroscopy.

With image guided navigation, radiation exposure can be up to one fourth of radiation with conventional radiology (29). Also the initial radiographs (mounting radiographs for the navigation unit) can be taken while both the surgeon and the operating room personnel are standing away from the source of radiation. After that, the source of radiation can be removed and the surgeon and his assistants can come close to the patient with no extra radiation needed. It is known that the radiation-induced cancer risk is cumulative, what places surgical staff under real risk $(13,19,24,30-37)$.

Also, CAOS decreases the number of false passages. In conventional fluoroscopy, the guide wire is moved forward while being viewed in one plane and then its position is checked in the other needed plans with the $\mathrm{C}$-arm. If the direction of the guide wire is incorrect, it will create a false passage. With CAOS, the procedure is done with the imaginary guide wire (trajectory) in all four planes on the navigation monitor before advancing the guide wire, assuring that the guide wire is in the correct corridor before drilling it.

\section{There are certain obstacles to widespread use of navigation in trauma practice}

It can be used only in fractures that are stable and where the bone ends do not move in relation to each other (if it is to be used in an unstable fracture, both fracture ends have to be traced by trackers). Again, bending of the guide wire may be responsible for disparity between the real position of tip of the guide wire and what is shown on the monitor of the navigation. This has been described as a possible cause of the inaccurate position of screws inserted by navigation in pelvic trauma surgery $(18,19)$. To avoid this problem, use of thicker wire or navigating solid drills (and using solid non-cannulated screws), rather than guide wires, is recommended.

There is a learning curve for using navigation in trauma. The position of the trackers should be where the pathways between them and the navigation unit is not blocked by the $\mathrm{C}$ arm, the surgeon, surgical instruments or (with obese patients) abdominal folds. Each time the pathway between the trackers and the navigation unit is interrupted, the picture on the computer screen will disappear, which may cause some frustration and delay. Navigation requires extra steps for set up in the operating room (insertion of tracking and registration), which will increase the surgery time at first when using this technology. With repeated use of navigation, the surgeon and operating room personnel may become more acquainted with these steps and the set up of navigation may cause a minimal increase in surgery time.

CAOS increases the cost of surgery. This may be the main obstacle against widespread use of navigation. There is a significant cost associated with CAOS, the initial hard- and software cost between 200,000 - 250,000 US dollars, followed by the cost of maintenance and software updating.

\section{Conclusion}

CAOS is becoming more popular in orthopedic trauma surgery, especially pelvic and acetabular fixation because of their complex anatomical nature and the need for extensive use of radiographs. It has positive effects that will benefit both patients and surgeons. It is an emerging technology and its use will expand tremendously with time, despite the current limitations. Orthopedic surgeons should be aware of CAOS and its development as it is expected to become more userfriendly and more affordable in future. 
Conflict of interest: The authors declare that they have no conflict of interest. This study was not sponsored by any external organization.

Authors' contributions: The three authors (AA, EK, RA) contributed to the conception and design, acquisition, analysis, interpretation of data, drafting the article and revising it critically for important intellectual content.

\section{References}

1. Biasca N, Schneider TO, Bungartz M. Minimally invasive computer-navigated total knee arthroplasty. Orthop Clin North Am. 2009;40(4):537-63.

2. Dattani R, Patnaik S, Kantak A, Tselentakis G. Navigation knee replacement. Int Orthop. 2009;33(1):7-10.

3. Krackow KA, Phillips MJ, Bayers-Thering M, Serpe L, Mihalko WM. Computer-assisted total knee arthroplasty: navigation in TKA. Orthopedics. 2003;26(10):1017-23.

4. Beckmann J, Stengel D, Tingart M, Götz J, Grifka J, Lüring C. Navigated cup implantation in hip arthroplasty. Acta Orthop. 2009;80(5):538-44.

5. Lützner J, Krummenauer F, Wolf C, Günther KP, Kirschner S. Computer-assisted and conventional total knee replacement: a comparative, prospective, randomised study with radiological and CT evaluation. J Bone Joint Surg Br. 2008;90(8):1039-44.

6. Pitto RP, Mueller LA, Reilly K, Schmidt R, Munro J. Quantitative computer-assisted osteodensitometry in total hip arthroplasty. Int Orthop. 2007;31(4):431-8.

7. Kalteis T, Handel M, Herold T, Perlick L, Baethis $\mathrm{H}$, Grifka J. Greater accuracy in positioning of the acetabular cup by using an image-free navigation system. Int Orthop. 2005;29(5):272-6.

8. Ishikawa $\mathrm{Y}$, Kanemura T, Yoshida G, Ito Z, Muramoto A, Ohno S. Clinical accuracy of three-dimensional fluoroscopy-based computer-assisted cervical pedicle screw placement: a retrospective comparative study of conventional versus computer-assisted cervical pedicle screw placement. J Neurosurg Spine. 2010;13(5):606-11.

9. Schlenzka D, Laine T, Lund T. Computer-assisted spine surgery. Eur Spine J. 2000;9(Suppl 1):S57-64.

10. Kahler DM. Navigated long-bone fracture reduction. J Bone Joint Surg Am. 2009;91(Suppl 1):102-7.

11. Chao EYS, chair. NIH/AAOS research symposia: new engineering technology transfer in orthopaedics; 1998 Apr 30 - May 3; Baltimore, Maryland, USA.
12. Kanlić EM, Delarosa F, Pirela-Cruz M. Computer assisted orthopaedic surgery -- CAOS. Bosn J Basic Med Sci. 2006;6(1):7-13.

13. Atesok K, Schemitsch EH. Computer-assisted trauma surgery. J Am Acad Orthop Surg. 2010;18(5):247-58.

14. Stöckle U, Schaser K, König B. Image guidance in pelvic and acetabular surgery--expectations, success and limitations. Injury. 2007;38(4):450-62.

15. Kendoff D, Ortega G, Citak M, Citak M, Hüfner T, Krettek C, Olivier LC. Limitations and pitfalls of 3-D fluoroscopic navigation in orthopaedic trauma surgery. Technol Health Care. 2009;17(2):133-40.

16. Barrick EF, O'Mara JW, Lane HE 3rd. Iliosacral screw insertion using computer-assisted CT image guidance: a laboratory study. Comput Aided Surg. 1998;3(6):289-96.

17. Hüfner T, Gebhard F, Grützner PA, Messmer P, Stöckle U, Krettek C. Which navigation when? Injury. 2004;35(Suppl 1):S-A30-4.

18. Rosenberger RE, Dolati B, Larndorfer R, Blauth M, Krappinger D, Bale RJ. Accuracy of minimally invasive navigated acetabular and iliosacral fracture stabilization using a targeting and noninvasive registration device. Arch Orthop Trauma Surg. 2010;130(2):223-30.

19. Arand M, Kinzl L, Gebhard F. Computer-guidance in percutaneous screw stabilization of the iliosacral joint. Clin Orthop Relat Res. 2004;(422):201-7.

20. Matta J, Saucedo T. Internal fixation of pelvic ring fractures. Clin Orthop. 1989;242:83-97.

21. Parker PJ, Copeland C. Percutaneous fluoroscopic screw fixation of acetabular fractures. Injury. 1997;28(9-10):597-600.

22. Routt Jr ML, Kregor PJ, Simonian PT, Mayo KA. Early results of percutaneous ilisacral screws placed with the patient in the supine position. J Orthop Trauma. 1995;9(3):207-14.

23. Schep NW, Haverlag R, van Vugt AB. Computer assisted versus conventional surgery for insertion of 96 cannulated iliosacral screws in patients with postpartum pelvic pain. J Trauma. 2004;57(6):1299-302.

24. Tonetti J, Carrat L, Blendea S, Merloz P, Troccaz J, Lavallée $S$, et al. Clinical results of percutaneous pelvic surgery. Computer assisted surgery using ultrasound compared to standard fluoroscopy. Comput Aided Surg. 2001;6(4):204-11.

25. Mosheiff R, Khoury A, Weil Y, Liebergall M. First generation computerized fluoroscopic navigation in percutaneous pelvic surgery. J Orthop Trauma. 2004;18:106-11.

26. Routt ML Jr, Nork SE, Mills WJ. Percutaneous fixation of pelvic ring disruptions. Clin Orthop Relat Res. 2000;(375):15-29. 
27. Crowl AC, Kahler DM. Closed reduction and percutaneous fixation of anterior column acetabular fractures. Comput Aided Surg. 2002;7(3):169-78.

28. Starr AJ, Reinert CM, Jones AL. Percutaneous fixation of the columns of the acetabulum: a new technique. J Orthop Trauma. 1998;12(1):51-8.

29. Hamelinck HK, Haagmans M, Snoeren MM, Biert J, van Vugt AB, Frölke JP. Safety of computer-assisted surgery for cannulated hip screws. Clin Orthop Relat Res. 2007;455:241-5.

30. Ebraheim NA, Coombs R, Jackson WT, Rusin JJ. Percutaneous computed tomography-guided stabilization of posterior pelvic fractures. Clin Orthop. 1994;307:222-8

31. Kraus MD, Krischak G, Keppler P, Gebhard FT, Schuetz UH. Can computer-assisted surgery reduce the effective dose for spinal fusion and sacroiliac screw insertion? Clin Orthop Relat Res. 2010;468(9):2419-29.

32. Smith GL, Briggs TWR, Nordeen H. Ionising radiation: are orthopaedic surgeons at risk? Ann R Coll Surg Engl. 1992;74:326-8.
33. Smith-Bindman R, Lipson J, Marcus R, Kim KP, Mahesh M, Gould R, et al. Radiation dose associated with common computed tomography examinations and the associated lifetime attributable risk of cancer. Arch Intern Med. 2009;169(22):2078-86.

34. Singh, PJ, Perera NS, Dega R. Measurement of the dose of radiation to the surgeon during surgery to the foot and ankle. Bone Joint Surg. 2007;89B:1060-3.

35. Mastrangelo G, Fedeli U, Fadda E, Giovanazzi A, Scoizzato L, Saia B. Increased cancer risk among surgeons in an orthopaedic hospital. Occup Med (Lond). 2005;55(6):498-500.

36. Zwingmann J, Konrad G, Kotter E, Südkamp NP, Oberst M. Computer-navigated iliosacral screw insertion reduces malposition rate and radiation exposure. Clin Orthop Relat Res. 2009;467(7):1833-8.

37. Collinge C, Coons D, Tornetta P, Aschenbrenner J. Standard multiplanar fluoroscopy versus a fluoroscopically based navigation system for the percutaneous insertion of iliosacral screws: a cadaver model. J Orthop Trauma. 2005;19(4):254-8. 